\title{
Les congrès en l'an 2030 !
}

\section{Congresses in 2030!}

\author{
G. Lesur \\ (C) Springer-Verlag France 2014
}

Pierre Dac avec son humour légendaire disait « Les prédictions sont difficiles... surtout lorsqu'elles concernent l'avenir ». À quelques jours du grand congrès annuel francophone de pathologie digestive, risquons-nous à réfléchir à ce que pourraient être les congrès médicaux en 2030. Car hormis le sexe des participants qui y sera très certainement majoritairement féminin... beaucoup d'inconnues persistent ! « Mais nous n'y serons plus » penseront les plus âgés d'entre vous, cependant qui sait si l'âge de la retraite ne va pas encore être reculé ?

Le mot congrès vient du latin « congressum » et signifie " réunion de personnes qui délibèrent sur des recherches, des études communes ou des intérêts communs en différents domaines ». Les congrès dans leur forme actuelle rentrent naturellement dans ce cadre avec cette notion de réunion, c'est-à-dire de rassemblement de personnes. Mais les hommes du dictionnaire étaient prévoyants puisque rien n'indique que ces personnes aux centres d'intérêt communs soient nécessairement réunies dans un même endroit. Quant au moment ? Vous me voyez venir...

Sans être ni devin ni Cassandre, on peut sans grand risque penser que les congrès dans leur forme actuelle seront bientôt du passé et il y a à cela plusieurs raisons. D'abord, des raisons financières car le coût de l'inscription à ces congrès augmente alors même que chacun est de plus en plus souvent contraint de régler sa participation avec ses propres deniers. Et comme cela est un fait nouveau, on peut comprendre qu'il y ait, sur ce point, quelques grincements de dents et de la résistance. Et ce d'autant qu'il n'y a pas beaucoup de professions qui se forment aussi régulièrement et consciencieusement que la profession médicale. L'obligation de formation qui nous est faite pourrait, comme dans d'autres domaines, valoir compensation. Mais ce combat n'est pas à mener, car dans les circonstances actuelles, il est perdu d'avance.
Mais si coût nouveau il y a pour nous médecins, coût croissant il y a aussi pour les organisateurs. Pour ces organisateurs qui pourraient voir progressivement diminuer le nombre de participants, une façon de réduire les coûts est naturellement de ne plus faire de réunion physique... mais avec le risque pour ces mêmes organisateurs de disparaître. Surtout à ces freins financiers pourraient venir s'ajouter des "accélérateurs » techniques. La technique moderne permet chaque jour un peu plus et un peu mieux dans le domaine des transmissions, des échanges et du partage d'informations qui sont le cœur et le nœud fédérateur des réunions physiques actuelles. Et l'exemple de certaines réunions post-congrès organisées sous forme de vidéoconférences interactives va certainement se développer. Ce n'est donc pas de la science-fiction que d'imaginer qu'il en sera de même pour la plupart des congrès médicaux dans les années à venir. Dans un autre domaine, qui aurait imaginé il y a seulement dix ans que tous les concerts de l'Orchestre Philharmonique de Berlin sont désormais visibles par tous 24 heures sur 24 sur Internet ? Car si le congrès dans sa forme actuelle réunit dans un même lieu, il le fait également dans un temps donné. À l'avenir, il est plus que probable que ces deux éléments, lieu et temps, soient dissociés, permettant à chacun de se former comme il l'entend.

En attendant le futur où vous vous mettrez à niveau sur le traitement minute d'Helicobacter pylori ou la guérison des cancers coliques par vidéocapsule chargée de molécules anticancéreuses efficaces, tout en commandant votre micro-onde et en téléchargeant (gratuitement ?) les derniers tubes de Stromae depuis votre montre/smartphone/CB/code génétique, c'est pour moi un grand plaisir que de vous permettre grâce à ce numéro d'Acta Endoscopica d'avoir de nouveau accès à une version papier des résumés des communications de ces JFHOD consacrées à l'endoscopie. Car comme disait Pierre Dac, « l'avenir, c'est du passé en préparation ». Bonnes JFHOD 2014 et bonne lecture !

G. Lesur $(\bowtie)$

Rédacteur en chef, hôpital Ambroise Paré, F-92100 Boulogne

e-mail : gilles.lesur@apr.aphp.fr 